\title{
A Study to Find out the Relationship between Various Platelet Indices and Morbidity Profile of Dengue Fever in Pediatric Patients on Admission in Western Rajasthan, India
}

\author{
Mohan Makwana ${ }^{1}$, Sangeeta Kumari ${ }^{2}$, Harish K Mourya ${ }^{3}$, Ramavtar Mitharwal ${ }^{4}$, Shivji Ram ${ }^{5}$
}

\begin{abstract}
AbStARCt
Background: Dengue is a major international public health concern. Various clinical and biochemical parameters are being used to predict severity of this disease. Blood platelets and their indices are one of them. Platelet indices like mean platelet volume (MPV), platelet distribution width (PDW), and platelet large cell ratio (P-LCR) have been investigated as prospective platelet activation indicators.

Aims and objectives: To find out the relationship between the platelet indices at admission and morbidity profile of dengue patients. Materials and methods: The study was an observational study conducted on patients of pediatric age group in Department of Pediatrics, Dr SN Medical College, Jodhpur, over a period of 1 year. All ELISA (lgM + IgG) positive cases were enrolled in the study.

Results: In this study high MPV, high PDW, and high P-LCR were associated with longer duration of hospital stay and longer duration of fluid therapy requirement. These patients required ICU care more frequently, more time to recover from severe thrombocytopenia (TCP), and had deranged SGPT and serum creatinine values, which were commonly seen with high MPV. Shock was more frequently seen with high MPV, and bleeding manifestations were more common with low MPV. Almost $80 \%$ patients were admitted with low plateletcrit (PCT), which was associated with longer duration of hospital stay, longer duration of fluid therapy, required ICU care more frequently, required more time to recover from severe TCP, landed up in shock more frequently, and required more blood product transfusion. High MPV, high P-LCR, low PCT, and severe TCP were associated with more frequent positive tourniquet test with a highly significant $p$ value. Bleeding manifestations and neurological symptoms were seen more with low MPV.

Conclusion: We observed significant correlation of P-LCR, PDW, and MPV on admission with those morbidity parameters. High MPV, high P-LCR, high PDW, and low PCT were associated with longer duration of hospital stay, fluid therapy requirement, time taken to recover from TCP, frequent requirement of ICU care, and more frequent liver and renal function dysfunctions. They had higher chances of developing dengue shock syndrome.
\end{abstract}

Keywords: Dengue fever, MPV, PDW, Platelet indices, Plateletcrit, PLCR.

Pediatric Infectious Disease (2020): 10.5005/jp-journals-10081-1252

\section{INTRODUCTION}

Dengue fever (DF) is the fastest emerging arboviral infection of major public health consequences for millions of people around the world, and in particular the Southeast Asia and Asia-Pacific regions of the World Health Organization (WHO). ${ }^{1}$ In order to elucidate the usefulness of various tests in the early course of dengue infection, in terms of diagnosis and correlation with clinical severity, people have used various clinical and laboratory markers. In this regards, decreasing platelet counts have been found to predict the severity of the disease. Platelet indices like mean platelet volume (MPV), platelet distribution width (PDW), and platelet large cell ratio (P-LCR) have been investigated as prospective platelet activation indicators. ${ }^{2}$ MPV is a useful independent predictor of bleeding. It is surrogate indicator of bone marrow activity; a high MPV is suggestive of increased megakaryocyte activity. A low MPV indicates bone marrow suppression and suggests a risk of bleeding. Correlation of parameters like platelet count and MPV with bleeding and severity of the disease can be a predictor of disease outcome. ${ }^{3}$

Platelet distribution width is a marker of platelets volume variability and is elevated in the presence of platelet anisocytosis. PDW directly measures variability in platelet size, changes occurring with platelet activation, and suggests the heterogeneity in platelet morphology. ${ }^{4-9}$

Plateletcrit (PCT) is a measurement of total platelet mass. Plateletcrit is useful for detecting platelet quantitative abnormalities. It is the volume occupied by platelets in the blood as a percentage.
${ }^{1-5}$ Department of Pediatrics, Dr SN Medical College, Jodhpur, Rajasthan, India

Corresponding Author: Mohan Makwana, Department of Pediatrics, Dr SN Medical College, Jodhpur, Rajasthan, India, Phone: +91 9414127916, e-mail: mohanmakwana32@yahoo.com

How to cite this article: Makwana M, Kumari S, Mourya HK, et al. A Study to Find out the Relationship between Various Platelet Indices and Morbidity Profile of Dengue Fever in Pediatric Patients on Admission in Western Rajasthan, India. Pediatr Inf Dis 2020;2(2):43-50.

Source of support: Nil

Conflict of interest: None

These markers have been used solely or in combination to predict the severity in dengue infections. We planned this study to evaluate platelets profile (platelet indices) and understand its importance (in terms of their values on admission and its relationship with the morbidity profile) so that adverse outcomes of this rapidly spreading life-threatening disease can be predicted early.

\section{Aims and OBjectives}

To find out the relationship between the platelet indices at admission and morbidity profile of dengue patients. 


\section{Outcome Measures}

The morbidity profile of these patients is based on their platelet indices at admission.

\section{Materials and Methods}

It is an observational study conducted on patients of dengue of pediatric age group in Department of Pediatrics, Dr SN Medical College, Jodhpur, over a period of 1 year from January 2018 to December 2018.

\section{Inclusion Criteria}

Eligible patients fulfilling diagnostic criteria as per WHO were enrolled and were followed up during their hospitalization.

\section{Exclusion Criteria}

Following patients were excluded from the study:

- Not giving written consent

- Patient who had proven other cause of thrombocytopenia (TCP)

- Having other diagnosed cause of fever

A written consent was taken from the parent/guardian; permission from the ethical committee was obtained for the study.

All children were managed as per standard protocols.

All statistical analyzes were performed by using the SPSS 24.0 software package (SPSS Inc., Chicago, IL, USA). The Yates continuity correction test (Chi-square test), the Fisher's exact test, and the Fisher-Freeman-Halton test were used for comparison of qualitative data, and the Student's $t$-test was summarized as mean $\pm S D$ for continuous variables, numbers, and percentages for categorical variables. A $p<0.05$ was accepted as statistically significant. The Chi-square test was used for categorical data. The confidence interval taken as $95 \%$. For good discrimination, the area under curve (AUC) was taken as more than 0.7.

\section{Results}

The present study was conducted in a tertiary care center of Western Rajasthan. During the study period, out of total indoor patient's department (IPD) of 49,711 patients were screened for dengue and 1,750 were detected positive for NS-1 by rapid diagnostic tests. Out of these, a total of 210 cases were confirmed positive by ELISA (IgM and IgG). These 210 cases were enrolled in the study and investigated as per study protocols (Flowchart 1). The salient observations made from the study were as follows:

In our study, maximum number $(128 ; 60.95 \%)$ of cases were detected in the month of October followed by November (37; $17.62 \%$ ), while no case were found in months of April, May, and June. Maximum number of patients were males (55.23\%) as compared to females (44.76) with a male female ratio of 1.2:1. Almost all patients (99.52\%) presented with TCP, out of which $34.2 \%$ were with severe, $29.52 \%$ with moderate, and $34.76 \%$ with mild TCP on day 1 of admission. Only three (1.42\%) patients were admitted with the normal range of platelet counts (Table 1 ).

Most of the cases (84.76\%) required average stay in the hospital for less than a week; nearly $2 \%$ of the patients stayed for more than 2 weeks. The mean duration of hospital stay was $4.16 \pm 2.52$ days.

In our study, 22\% patients required ICU care. Out of the total cohort, $83.81 \%$ patients improved without developing the shock, while $16.19 \%$ patients landed up in shock; $18 \%$ patients required blood products. Mean duration to achieve platelet count above or equal to 1 lac was found to be $3.41 \pm 2.60$ days.

We observed that there is no significant variation in the hemoglobin of patients who were IgM plus IgG positive in comparison with those who were only lgM positive having mean values of $12.77 \pm 1.84$ and.12.73 \pm 1.64 ; similarly, mean HCT of patients who were both $\mathrm{lgM}$ and IgG positive was found to be 35.96 \pm 4.40 and mean HCT of $36.36 \pm 4.10$ was found in patients who were IgG negative. Total leukocyte count (TLC) was almost similar in both the groups. However, PC (64.91 \pm 43.00$),$ MPV (10.32 \pm 2.24$)$, and PLCR (25.76 \pm 9.65$)$ were observed higher in IgG-negative patients, which was statistically significant (Table 2).

We observed moderate TCP on 1st day of admission, which was seen in almost all patients irrespective of their age, but maximum severity was seen in the age group 10-18 years with a mean platelet count of $71.85 \pm 45.05$ on day 1 of admission (Table 3 ).

High MPV was associated with longer duration of hospital stay with a mean value of $5.80 \pm 2.38$ days, longer duration of fluid therapy requirement ( $3.60 \pm 1.51$ days), required ICU care more frequently $(66.66 \%)$, more commonly associated with shock (66.66\%), and required more time to recover from severe TCP. All patients with high MPV were associated with deranged SGPT levels; similarly deranged serum creatinine values were commonly seen with high MPV. Low MPV was associated with bleeding manifestations and neurological symptoms more frequently (52.63 and $39.47 \%$, respectively) (Table 4 ).

Maximum patients enrolled in our study were having normal PDW (85.7\%) but patients who were having high PDW (7.15\%) were stayed in hospital for longer duration ( $6.40 \pm 4.23$ days), required ICU care more frequently (53.33\%), required blood products more commonly (46.66\%), and have taken more time to recover from $\mathrm{TCP}(4.26 \pm 2.01$ mean days). Both neurological symptoms and bleeding manifestations were seen higher with high PDW with a frequency of 60 and $100 \%$, respectively ( $p$ value 0.002 and $<0.0001$, respectively) (Table 5 ).

In our observations, hospital stay $(4.34 \pm 2.66)$ and fluid therapy $(2.25 \pm 1.27)$ given in days were seen longer with low PCT values. The patients with low PCT landed up in shock more frequently (20.24\%) and were admitted in ICU more commonly (25\%), were given blood products in $18.45 \%$ of them, and they took more time to recover from TCP. The patients with low PCT were associated more commonly with deranged liver function tests (LFT), more frequent neurological symptoms, and bleeding manifestations (Table 6).

Table 7 shows that $68 \%$ patients were admitted with normal P-LCR, while $24.76 \%$ were seen with high P-LCR. Only $7.14 \%$ were admitted with low P-LCR values. High P-LCR was associated with longer duration of hospital stay and longer duration of fluid therapy requirement with mean values of $4.96 \pm 3.26$ ( $p$ value 0.022 ) and $2.34 \pm 1.18$ ( $p$ value 0.289 ). Maximum patients who received ICU care belonged to the normal P-LCR group (28 patients; $19.58 \%$ ) but out of total patients who were admitted with high P-LCR. High P-LCR was more frequently associated with neurological symptoms, bleeding manifestations, and blood product transfusion.

We observed that those patients who were tourniquet test positive were found to have high MPV with a mean value of 10.97 \pm 2.84 , low PCT with a mean value $0.07 \pm 0.05$, high P-LCR with a mean value of $33.07 \pm 9.94$, and low platelet counts with a mean value of $34.72 \pm 35.62 \times 10^{3} / \mu \mathrm{L}$ (Table 8). 
Flowchart 1: Flowchart of the observational study

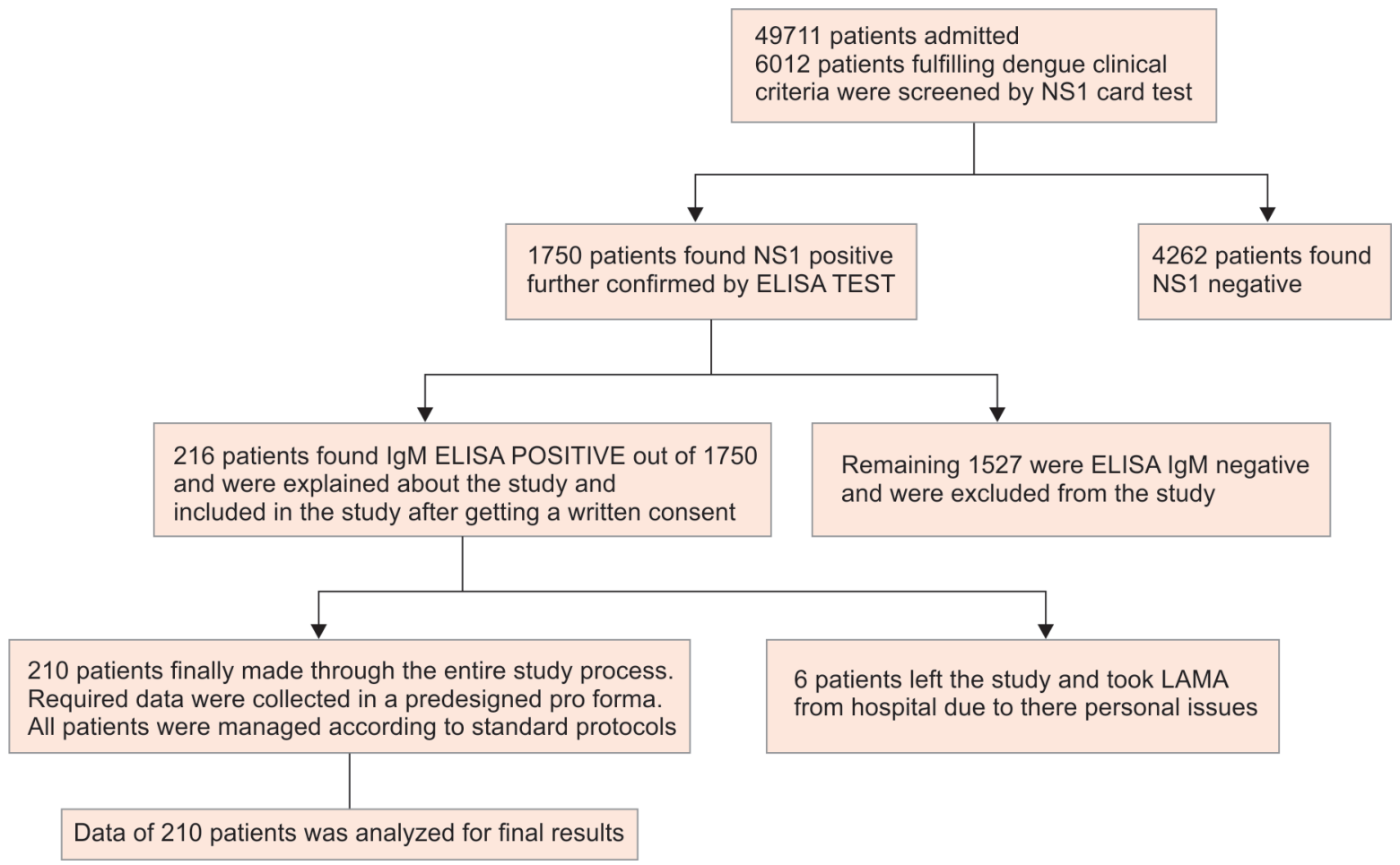

Table 1: Platelet count in dengue cases

\begin{tabular}{lccccc}
\hline $\begin{array}{l}\text { Platelet count per } \\
\text { microliter }\end{array}$ & Day 1 & Day 2 & Day 3 & Day 4 & Day 5 \\
\hline$<10,000$ & $20(9.52 \%)$ & $5(2.45 \%)$ & $3(1.85 \%)$ & - & - \\
$10,000-50,000$ & $52(24.7 \%)$ & $42(20.59 \%)$ & $17(10.49 \%)$ & $6(8.22 \%)$ & $4(28.57 \%)$ \\
$50,000-100,000$ & $62(29.52 \%)$ & $71(34.80 \%)$ & $46(28.40 \%)$ & $17(23.29 \%)$ & $1(7.14 \%)$ \\
$100,000-150,000$ & $73(34.76 \%)$ & $36(17.65 \%)$ & $46(28.40 \%)$ & $29(39.73 \%)$ & $6(42.86 \%)$ \\
$>150,000$ & $3(1.42 \%)$ & $50(24.51 \%)$ & $50(30.86 \%)$ & $21(28.77 \%)$ & $3(21.43 \%)$ \\
Total & $210(100 \%)$ & $204(97.14 \%)$ & $162(77.14 \%)$ & $73(34.76 \%)$ & $14(6.67 \%)$ \\
\hline
\end{tabular}

Table 2: ELISA IgM and IgG test findings with other parameters at admission

\begin{tabular}{|c|c|c|c|}
\hline \multirow[b]{2}{*}{$\begin{array}{l}\text { Laboratory param- } \\
\text { eters }\end{array}$} & \multicolumn{2}{|c|}{ ELISA test results } & \multirow[b]{2}{*}{$p$ value } \\
\hline & $\begin{array}{l}\lg M+\lg G \\
\text { positive }(n=68)\end{array}$ & $\begin{array}{l}\text { IgG negative } \\
(n=142)\end{array}$ & \\
\hline $\mathrm{Hb}(\mathrm{g} / \mathrm{dL})$ & $12.77 \pm 1.84$ & $12.73 \pm 1.64$ & 0.847 \\
\hline $\mathrm{HCT}(\%)$ & $35.96 \pm 4.40$ & $36.36 \pm 4.10$ & 0.518 \\
\hline $\operatorname{TLC}\left(\times 10^{3} / \mu \mathrm{L}\right)$ & $4.35 \pm 3.01$ & $4.43 \pm 3.09$ & 0.863 \\
\hline $\mathrm{PC}\left(\times 10^{3} / \mu \mathrm{L}\right)$ & $64.91 \pm 43.00$ & $82.08 \pm 40.29$ & 0.005 \\
\hline MPV (fL) & $10.32 \pm 2.24$ & $11.07 \pm 1.91$ & 0.013 \\
\hline РCT (\%) & $0.14 \pm 0.11$ & $0.14 \pm 0.08$ & 0.979 \\
\hline $\begin{array}{l}\text { Platelet-large cell } \\
\text { ratio (PT-LCR) (\%) }\end{array}$ & $25.76 \pm 9.65$ & $29.40 \pm 8.77$ & 0.006 \\
\hline $\begin{array}{l}\text { Platelet distribution } \\
\text { width (PDW) (\%) }\end{array}$ & $11.97 \pm 3.09$ & $12.81 \pm 3.26$ & 0.078 \\
\hline
\end{tabular}

When we classified patients of DF according to severity as per WHO, we observed that $72 \%$ patients were presented with DF alone, while almost $12 \%$ patients developed dengue hemorrhagic fever
(DHF) anytime during the course of hospitalization and $16.19 \%$ patients developed dengue shock syndrome (DSS) any time during hospitalization.

Table 9 shows that MPV was within normal range in all the three groups (IIII, III) with mean values of $10.29 \pm 1.56,10.90 \pm 2.45$, and $10.79 \pm 3.35$, respectively. Plateletcrit was found to be low in all three groups with lowest being in group III with mean value of $0.06 \pm 0.05$. PDW and P-LCR were with in normal limits in all three groups. HCT was seen normal in all three groups but found to be increasing as the severity increased, highest being in group III. Thrombocytopenia was found in all three groups with lowest values in group III having a mean value of $22.26 \pm 30.70$.

We observed that mean duration of hospital stay was maximum (7.58 \pm 2.54 days) in DSS group as compared to other two. All patients with DSS were kept in ICU and $83 \%$ of total DSS patients required blood product transfusion. Time taken to recover from TCP and duration of fluid therapy requirement was also more with the DSS group with mean values of $6.11 \pm 2.07$ and $3.88 \pm 1.06$, respectively. Other system involvement was also more frequent with the DSS group (Table 10). 
Table 3: Correlation between age and platelet counts on first day of admission

\begin{tabular}{llllllr}
\hline \multirow{2}{*}{$\begin{array}{l}\text { Age group (in } \\
\text { years) }\end{array}$} & \multicolumn{5}{c}{ Platelet counts $\times 10^{3} / \mu \mathrm{L}$} \\
\cline { 2 - 6 } & Day 1 & Day 2 & Day 3 & Day 4 & Day 5 & ANOVA \\
\hline $1-4$ & $79.87 \pm 39.45$ & $112.48 \pm 67.92$ & $127.86 \pm 73.32$ & $141.84 \pm 49.87$ & $150.25 \pm 83.54$ & 0.0009 \\
$5-9$ & $75.58 \pm 42.62$ & $114.73 \pm 84.83$ & $132.62 \pm 92.86$ & $150.70 \pm 150.28$ & $114.50 \pm 15.71$ & 0.0002 \\
$10-18$ & $71.85 \pm 45.05$ & $109.43 \pm 79.47$ & $128.18 \pm 97.58$ & $137.76 \pm 131.72$ & $82.50 \pm 46.30$ & $<0.0001$ \\
\hline
\end{tabular}

Table 4: MPV values with various morbidity parameters

\begin{tabular}{|c|c|c|c|c|}
\hline \multirow[b]{2}{*}{ Parameters } & \multicolumn{4}{|c|}{$M P V(f L)$} \\
\hline & $\begin{array}{l}\text { Normal, } 8.60-15.60, \\
(n=166)(79 \%)\end{array}$ & $\begin{array}{l}\text { Low, }<8.60 \\
(n=38)(18 \%)\end{array}$ & $\begin{array}{l}\text { High, }>15.60, \\
(n=6)(3 \%)\end{array}$ & $\begin{array}{l}\text { ANOVA, } \\
p \text { value }\end{array}$ \\
\hline Average hospital stay (days) & $3.95 \pm 2.61$ & $4.91 \pm 2.61$ & $5.80 \pm 2.38$ & 0.037 \\
\hline Fluid therapy requirement (days) & $2.19 \pm 1.38$ & $3.24 \pm 1.49$ & $3.60 \pm 1.51$ & $<0.0001$ \\
\hline ICU care & $31(18.67 \%)$ & $20(52.63 \%)$ & $4(66.66 \%)$ & $<0.0001$ \\
\hline Shock & $27(16.26 \%)$ & $3(7.89 \%)$ & $4(66.66 \%)$ & 0.001 \\
\hline Blood products given & $20(12.04 \%)$ & $16(42.10 \%)$ & $3(50 \%)$ & $<0.0001$ \\
\hline Time to recover from TCP $>100,000$ (days) & $2.84 \pm 2.35$ & $4.27 \pm 3.16$ & $5.00 \pm 3.31$ & 0.004 \\
\hline Liver dysfunction SGPT > 45 IU & $118(71.08 \%)$ & $13(34.21 \%)$ & $6(100 \%)$ & $<0.0001$ \\
\hline RFT creatinine $>1.0 \mathrm{mg} \%$ & $24(14.45 \%)$ & $4(10.52 \%)$ & $2(33.33 \%)$ & 0.329 \\
\hline Neurological symptoms & $34(20.48 \%)$ & $15(39.47 \%)$ & $1(16.67 \%)$ & 0.042 \\
\hline Bleeding manifestation & $33(19.88 \%)$ & 20 (52.63\%) & $1(16.67 \%)$ & 0.0001 \\
\hline
\end{tabular}

Table 5: PDW values with various morbidity parameters

\begin{tabular}{|c|c|c|c|c|}
\hline \multirow[b]{2}{*}{ Parameters } & \multicolumn{4}{|c|}{ PDW (\%) } \\
\hline & $\begin{array}{l}\text { Normal, } 8.30-25.0 \\
(n=180)(85.71 \%)\end{array}$ & $\begin{array}{l}\text { Low, }<8.30(n=15) \\
(7.14 \%)\end{array}$ & $\begin{array}{l}\text { High, }>25.00 \\
(n=15)(7.14 \%)\end{array}$ & $p$ value \\
\hline Average hospital stay (days) & $4.02 \pm 2.30$ & $3.64 \pm 1.94$ & $6.40 \pm 4.23$ & 0.001 \\
\hline Fluid therapy given (days) & $2.38 \pm 1.48$ & $2.42 \pm 1.15$ & $2.80 \pm 1.56$ & 0.574 \\
\hline ICU care & $45(22.45 \%)$ & $2(14.29 \%)$ & $8(53.33 \%)$ & 0.028 \\
\hline Shock & $33(16.84 \%)$ & $1(7.14 \%)$ & $1(6.6 \%)$ & 0.283 \\
\hline Blood products given & $30(15.31 \%)$ & $1(7.14 \%)$ & $7(46.66 \%)$ & 0.007 \\
\hline Time to recover from TCP $>100,000$ (days) & $3.11 \pm 2.63$ & $2.35 \pm 2.49$ & $4.26 \pm 2.01$ & 0.129 \\
\hline Liver dysfunction SGPT > $45 \mathrm{IU}$ & $130(66.33 \%)$ & $9(64.29 \%)$ & - & 0.374 \\
\hline RFT S. creatinine $>1.0 \mathrm{mg} \%$ & $27(13.78 \%)$ & $3(21.43 \%)$ & - & 0.707 \\
\hline Neurological symptoms & $39(21.67 \%)$ & $2(13.33 \%)$ & $9(60.0 \%)$ & 0.002 \\
\hline Bleeding manifestation & 37 (20.56\%) & $2(13.33 \%)$ & 15 (100.0\%) & $<0.0001$ \\
\hline
\end{tabular}

Table 6: PCT values with various morbidity parameters

\begin{tabular}{|c|c|c|c|c|}
\hline \multirow[b]{2}{*}{ Parameters } & \multicolumn{4}{|c|}{ PCT (\%) } \\
\hline & $\begin{array}{l}\text { Normal, } 0.22-0.24 \\
(n=18)\end{array}$ & $\begin{array}{l}\text { Low, }<0.22 \\
(n=168)\end{array}$ & $\begin{array}{l}\text { High, }>0.24 \\
(n=24)\end{array}$ & $p$ value \\
\hline Average hospital stay (days) & $3.27 \pm 0.89$ & $4.34 \pm 2.66$ & $3.58 \pm 2.18$ & 0.114 \\
\hline Fluid therapy given (days) & $1.88 \pm 0.83$ & $2.25 \pm 1.27$ & $1.29 \pm 1.04$ & 0.001 \\
\hline ICU care & $1(5.56 \%)$ & $42(25.0 \%)$ & $3(12.50 \%)$ & 0.082 \\
\hline Shock & 00 & $34(20.24 \%)$ & - & NA \\
\hline Blood products given & 00 & 31 (18.45\%) & - & NA \\
\hline Time to recover from TCP $>100,000$ (days) & $1.31 \pm 1.13$ & $2.09 \pm 1.59$ & $0.68 \pm 1.08$ & 0.0001 \\
\hline Liver dysfunction SGPT >45 IU & $10(55.56 \%)$ & $112(66.67 \%)$ & $17(70.83 \%)$ & 0.560 \\
\hline RFT S. creatinine $>1.0 \mathrm{mg} / \mathrm{dL}$ & $1(5.56 \%)$ & $24(14.29 \%)$ & $5(20.83 \%)$ & 0.375 \\
\hline Neurological symptoms & $2(11.11 \%)$ & $43(25.60 \%)$ & $5(20.83 \%)$ & 0.365 \\
\hline Bleeding manifestations & 000 & $53(31.55 \%)$ & $1(4.17 \%)$ & 0.0005 \\
\hline
\end{tabular}


Table 7: P-LCR values with various morbidity parameters

\begin{tabular}{|c|c|c|c|c|}
\hline \multirow[b]{2}{*}{ Parameters } & \multicolumn{4}{|c|}{$P-L C R(\%)$} \\
\hline & $\begin{array}{l}\text { Normal, } 15.0-35.0 \\
(n=168)(68 \%)\end{array}$ & $\begin{array}{l}\text { Low, }<15.0(n=15) \\
\text { (7.14\%) }\end{array}$ & $\begin{array}{l}\text { High, }>35.0 \\
(n=52)(24.76 \%)\end{array}$ & $p$ value \\
\hline Average hospital stay (days) & $3.95 \pm 2.21$ & $3.40 \pm 1.88$ & $4.96 \pm 3.26$ & 0.022 \\
\hline Fluid therapy given (days) & $2.03 \pm 1.26$ & $2.00 \pm 1.25$ & $2.34 \pm 1.18$ & 0.289 \\
\hline ICU care (no) & $28(19.58 \%)$ & $2(13.33 \%)$ & $16(30.77 \%)$ & 0.175 \\
\hline Shock (no) & $18(12.59 \%)$ & $1(6.67 \%)$ & $15(28.85 \%)$ & 0.014 \\
\hline Blood products given & $1(0.70 \%)$ & $1(6.67 \%)$ & $14(26.92 \%)$ & $<0.0001$ \\
\hline Time to recover from TCP $>100,000$ & $1.82 \pm 1.64$ & $1.40 \pm 1.72$ & $2.16 \pm 1.29$ & 0.210 \\
\hline Liver dysfunction SGPT >45 IU & $90(62.94 \%)$ & $10(6.67 \%)$ & $39(75.0 \%)$ & 0.289 \\
\hline RFT S. creatinine $>1.0 \mathrm{mg} \%$ & $16(11.19 \%)$ & $4(26.67 \%)$ & $10(19.23)$ & 0.132 \\
\hline Neurological symptoms & $34(20.24 \%)$ & $2(13.33 \%)$ & $14(26.92 \%)$ & 0.435 \\
\hline Bleeding manifestation & $19(13.19 \%)$ & $3(20.00 \%)$ & $32(61.54 \%)$ & $<0.0001$ \\
\hline
\end{tabular}

Table 8: Tourniquet test in relation with various platelet indices

\begin{tabular}{lllll}
\hline Tourniquet test & $M P V(f L)$ & $P C T(\%)$ & $P L C R(\%)$ & $P C\left(\times 10^{3} / \mu L\right)$ \\
\hline Positive & $10.97 \pm 2.84$ & $0.07 \pm 0.05$ & $33.07 \pm 9.94$ & $34.72 \pm 35.62$ \\
Negative & $10.28 \pm 1.75$ & $0.16 \pm 0.09$ & $26.74 \pm 8.44$ & $92.46 \pm 35.52$ \\
$p$ value & 0.040 & $<0.0001$ & $<0.0001$ & $<0.0001$ \\
\hline
\end{tabular}

Table 9: Distribution of various platelet indices and HCT values among three classes of DF

\begin{tabular}{lllllcc}
\hline Type & MPV (8.6-15.5) & $P C T(0.22-0.24)$ & $P D W(8.3-25)$ & $P T-L C R(15-35)$ & HCT (26-50) & $P C$ \\
\hline DF(I) & $10.29 \pm 1.56$ & $0.16 \pm 0.09$ & $12.10 \pm 3.34$ & $26.64 \pm 8.47$ & $37.38 \pm 3.40$ & $89.33 \pm 36.29$ \\
DHF(II) & $10.90 \pm 2.45$ & $0.09 \pm 0.07$ & $15.98 \pm 6.84$ & $31.43 \pm 10.58$ & $38.27 \pm 3.19$ & $50.87 \pm 40.79$ \\
DSS(III) & $10.79 \pm 3.35$ & $0.06 \pm 0.05$ & $15.81 \pm 5.46$ & $32.80 \pm 9.28$ & $39.64 \pm 4.02$ & $22.26 \pm 30.70$ \\
\hline Statistical analysis & & & & & & \\
\hline Test & MPV (8.6-15.5) & PCT (0.22-0.24) & $P D W(8.3-25.0)$ & $P T L C R(15.0-35.0)$ & HCT (26.0-50.0) & $P C$ \\
\hline I vs II & 0.001 & $* N A$ & 0.310 & 0.831 & 0.231 & $<0.0001$ \\
I vs III & 0.004 & $* N A$ & 0.0006 & 0.267 & $<0.0001$ & $<0.0001$ \\
II vs III & 0.787 & $* N A$ & 0.239 & 0.443 & 0.012 & 0.009 \\
\hline
\end{tabular}

${ }^{*} \mathrm{NA}$ means $p$ value could not calculate because one parameter $\mathrm{SD}=0.00$.

Table 10: Various morbidity parameters among three classes of DF

\begin{tabular}{|c|c|c|c|c|}
\hline Type & $D F(n=151)(72 \%)$ & $\operatorname{DHF}(n=25)(12 \%)$ & DSS $(n=34)(16 \%)$ & pvalue \\
\hline Hospital stay & $3.27 \pm 1.54$ & $4.95 \pm 3.16$ & $7.58 \pm 2.54$ & $<0.0001$ \\
\hline Fluid therapy requirement & $1.96 \pm 1.32$ & $3.16 \pm 1.16$ & $3.88 \pm 1.06$ & $<0.0001$ \\
\hline ICU care & $13(8.6 \%)$ & $9(36 \%)$ & $34(100 \%)$ & $<0.0001$ \\
\hline Blood products given & $4(2.6 \%)$ & $6(24 \%)$ & $28(82.3 \%)$ & $<0.0001$ \\
\hline Time to recover from TCP >100,000 & $2.26 \pm 2.14$ & $4.50 \pm 2.20$ & $6.11 \pm 2.07$ & $<0.0001$ \\
\hline Liver dysfunction SGPT >45 & $85(56.29 \%)$ & $21(84 \%)$ & $33(97 \%)$ & $<0.0001$ \\
\hline RFT creatinine $>1.0$ & $14(9.27 \%)$ & $9(36 \%)$ & $14(41.17 \%)$ & 0.0002 \\
\hline Neurological symptoms & $17(11.2 \%)$ & $6(24 \%)$ & $26(76.47 \%)$ & $<0.0001$ \\
\hline
\end{tabular}

\section{Discussion}

Maximum number of patients in our study were admitted during the period of October to December; our observations were similar to that of Rasul et al. ${ }^{10}$ They observed that dengue transmission occurred round the year but the peak occurred in post-monsoon seasons; similar findings were also observed by Tripathi et al. ${ }^{11}$ in 2008. Singla et al. ${ }^{12}$ observed that infection started in the postmonsoon season, with highest number of cases peaked in October (>70\%) and reduced till December every year.
In our study, we found that $80 \%$ enrolled patients were from urban areas in our study while Tripathi et al. in $2008^{11}$ observed that dengue transmission equally occurs in both urban and rural population. Bleeding is one of the dreaded complications of dengue infection having highly variable clinical presentations from simple skin/mucosal bleeds like petechiae, purpura, and epistaxis to severe bleeds like gastrointestinal bleeds and fatal intracranial bleeds. In our study, the overall incidence of the bleeding manifestations was seen in $32.85 \%$; the tourniquet test was positive in $22.8 \%$ of 
cases. Epistaxis (54.79\%), melena (24.65\%), and petechiae $(19.17 \%)$ were significant bleeding manifestations in enrolled cases. Other manifestation was hematemesis (1.36\%). Another study ${ }^{13}$ reported melena in $85.7 \%$, hematemesis in $9.5 \%$, and petechiae in $2.6 \%$ cases, whereas Agarwal et al. observed hematemesis in 39\%, epistaxis in $36 \%$, skin bleeds in $33 \%$, and tourniquet test positive in $32 \%$ cases. $^{14}$ Shivbalan et al. ${ }^{15}$ in his study of predictors of spontaneous bleeding reported that petechiae was the most frequent (46.6\%) followed by hematemesis $(26 \%)$, melena $(21.6 \%)$, and subconjunctival hemorrhage (6.6\%). Richard et al. ${ }^{16}$ found that the tourniquet test was positive in $100 \%$ cases, followed by petechiae $(43.5 \%)$ and epistaxis (39.1\%). There is poor sensitivity of the tourniquet test in the diagnosis of DHF. ${ }^{17}$

Bleeding manifestation was seen even above severe TCP $(>50,000)$ in our study. In these patients, the qualitative defect of platelets might be a contributory factor.

Our study revealed that patients who were having both IgM and IgG positive (possible secondary infection) had more severe TCP (64.91 \pm 43.00). They were having lower TLC (4.35 \pm 3.01$)$, lower MPV (10.32 \pm 2.24$)$, and lower PTLCR and PDW as compared to those who were found to be $\operatorname{lgM}$ positive only. The theory of immune enhancement, developed extensively by Halstead, ${ }_{17}^{17}$ predicts that individual who have been immunologically sensitized to one dengue virus serotype may develop nonneutralizing antibodies that actually enhance the entry of different serotypes of dengue viruses into mononuclear phagocytes, resulting in the activation of complement and kinins, and the release of mediators of vascular permeability. In a study from Sri Lanka by Malaviage et al. observed that secondary dengue infection increased the risk of severe disease in case of dengue. ${ }^{18}$

Chacko et al. ${ }^{19}$ observed that the mean hemoglobin level of the patients was $12.76 \mathrm{~g} / \mathrm{dL}$. In cases with DSS, it was more (13.4 $\mathrm{g} / \mathrm{dL})$ than in those without $(12.2 \% \mathrm{~g} / \mathrm{dL})$ and the difference was statistically significant $(p=0.0206)$. Average hematocrit was $37.68 \%$. In those with DSS, it was significantly more $(p=00.01352)$. Only 21 (28.77\%) patients had hematocrit levels of $40 \%$ or more of which 13 (61.9\%) had DSS. It was also found that 14/21 (66.67\%) (six children without and eight with DSS) were of the age 4-10 years with a hematocrit of $40 \%$.

We observed that most of the cases $(84.76 \%)$ required hospitalization for less than a week. Nearly $2 \%$ of the patients stayed for more than 2 weeks. The mean duration of stay was found to be $4.16 \pm 2.52$ days. Betty and Gayathri found that duration of hospital stay averaged 6.19 days with a maximum stay of 20 days and a minimum of 3 days. ${ }^{19}$

The WHO has categorized dengue into three groups, e.g., DF, DHF, and DSS. The incidence of DF in our study was $72 \%$. Out of 210 enrolled patients, DHF cases were $12 \%$ and DSS were $16 \%$. The incidence of DHF is comparable with study done by Chhina et al., ${ }^{20}$ which was $18.7 \%$, where the incidence of DHF in other study done by Itha ${ }^{21}$ et al. was $33 \%$. Kabra et al. ${ }^{22}$ reported DF (10\%), DHF $(42 \%)$, and DSS in (47\%) cases, but in our study DF was seen more commonly (72\%) as compared to DHF (12\%) and DSS (16\%).

The role of platelet and its indices has also been studied in neonatal sepsis and many other conditions like septic arthritis. Choudhary et al. ${ }^{23}$ observed that high PDW, high MPV, and low platelet count were more associated with neonatal sepsis.

In our study, high MPV on admission was associated with longer duration of hospital stay with a mean value of $5.80 \pm$ 2.38 days, longer duration of fluid therapy (3.60 \pm 1.51$)$, ICU care more frequently (66.66\%), more commonly associated with shock (66.66\%), and required more time to recover from severe TCP in our study. All patients with high MPV were associated with deranged SGPT levels; similarly, deranged serum creatinine values were commonly seen with high MPV. Low MPV $(<8.60 \mathrm{fL})$ was seen in $18.09 \%$ patients. The mean value of MPV was found to be $10.90 \pm$ 2.45 and $10.79 \pm 3.35$ in DF and DHF, respectively; similar findings were observed by Dewil, i.e., higher MPV indicated a higher large platelet count, which was a sign of increasing platelet turnover. The prevalence ratio of MPV enhancement $\geq 9.7 \mathrm{fL}$ was $4.8(95 \% \mathrm{Cl}$ $2.8-37.0 ; p<0.0001)$. The result indicated that MPV enhancement was a risk factor of worsening dengue infection. ${ }^{24}$ Gunawan $^{25}$ et al. found a negative correlation between MPV and platelet count $(r=-0.52 ; p<0.05)$, which suggested that the more severe dengue infection (more thrombocytopenic) will be positively correlated with higher MPV. This correlation of TCP with high MPV was explained by Maede ${ }^{26}$ et al. According to him, TCP induces the bone marrow to produce immature platelets with larger size, increasing MPV value. Kiran et al. ${ }^{27}$ found that the mean MPV values were $9.2 \mathrm{fL}$ in patients with platelet count below 20,000 as compared to $12 \mathrm{fL}$ in the platelet count group of $20,000-100,000$ and $13.8 \mathrm{fL}$ in patients with platelet count above 100,000 . A low MPV in Indian subset of patients has been reported in more than two-third of the Navya et al. study group of DF patients ${ }^{28}$ on contrary of which a case control study on role of platelet indices in patients with DF by Kanchana ${ }^{29}$ et al. found mean MPV of 7.29. Low MPV that indicates bone marrow suppression was noted in $36.19 \%$ of patients with DF and in $11.42 \%$ of patients with DHF they found that low platelet count, low MPV, and high PDW compared to normal may be used as probable indicators for dengue in endemic area. Low MPV $<9 \mathrm{fL}$ and high PDW $>13 \mathrm{fL}$ show considerable sensitivity for DF.

Kritika et al. observed that maximum number (61.11\%) of patients with normal MPV (8-12 fL MPV) presented with DSS as compared to those with low ( $<8 \mathrm{fL}$ ) MPV. Higher proportion $(55.15 \%)$ (75/136) of DF and $56.52 \%$ (26/46) of DHF cases were in >12 MPV range. ${ }^{30}$ However, no significant difference was observed in MPV with severity of disease. Anikethana ${ }^{31}$ et al. found no correlation between the platelet count, MPV, PDW, serology, gender, and severity of the cases. There was no significant correlation between the platelet count, MPV, and PDW.

Most of the patients enrolled in our study were having normal PDW (85.7\%). Mean PDW in DF, DHF, and DSS groups was observed to be $12.10 \pm 3.34,15.98 \pm 6.84,15.81 \pm 5.46$, respectively, but patients who were having high PDW (7.15\%) were associated with severe dengue, required longer duration of hospitalization $(6.40 \pm$ 4.23 days), required ICU care more frequently (53.33\%), given blood products more commonly (46.66\%), and have taken more time to recover from TCP $(4.26 \pm 2.01$ mean days); similar observations were made by Kanchana et al. according to which high PDW indicated as a useful marker for platelet activation and was seen in $59.19 \%$ of patients with DF and $11.42 \%$ in DHF patients ( $p$ value 0.001$){ }^{29}$

Kiran et al. found that platelet indices PDW ( $57 \pm 13.8$ vs 55.4 $\pm 6.9, p$ value 0.001$)$ and MPV $(9.2 \pm 0.09$ vs $13.8 \pm 1.3 \mathrm{fL}, p$ value $<$ 0.001 ) values were significantly altered in DF with platelet counts below 20,000 compared to platelet count more than 100,000 group. ${ }^{27}$

Prakash and Anikethana found no correlation between the platelet count, MPV, PDW, serology, gender, and severity of the cases. There was no significant correlation between the platelet count, MPV, and PDW. ${ }^{31}$ 
When we analyzed our data, we observed that hospital stay $(4.34 \pm 2.66)$ and fluid therapy $(2.25 \pm 1.27)$ given in days were seen longer with low PCT values. The patients with low PCT landed up in shock more frequently (20.24\%) and were admitted in ICU more commonly (25\%), were given blood products in $18.45 \%$ of them, and they took more time to recover from TCP. The patients with low PCT were associated more commonly with deranged LFT, more frequent neurological symptoms, and bleeding manifestations.

Chiranth et al. ${ }^{32}$ found that $64 \%$ patients were having PCT below $0.1 \%$, and $57 \%$ had a platelet count fall below 100,000 during their illness. Plateletcrit showed a significant correlation $(p=0.001)$ with decreasing and increasing trends of the platelet count and a similar relation to the severity of the disease. Patients had a longer duration of stay ( $>4$ days) when platelet counts decreased to less than 100,000 during their illness compared to those with more than $100,000$ (73\% vs $35 \%)$.

In a study from China by Xiaoyun ${ }^{33}$ et al., it was found that PLCR, PDW, and MPV were significantly higher in patients in acute febrile phase of DF, compared with the healthy subjects $(p<0.01)$, while PCT and PLT were significantly lower in patients in acute febrile phase of DF, compared with the healthy subjects $(p<0.01)$.

In our study, almost all patients presented with TCP. Severe TCP was noted in around $32 \%$ at admission. Minimum platelet count observed on admission was $2,000 / \mathrm{mm}^{3}$.

Rajesh et al. found that platelet counts at presentation were less than $50,000 / \mu \mathrm{L}$ in around $69.51 \%$ of cases, though it kept on falling further during hospitalization. Minimum platelet count noted was $8,000 / \mu \mathrm{L} .^{34}$

Navya et al. found that $24 \%$ of the total enrolled cases were having severe TCP, out of which $8 \%$ were having platelet count less than $20,000 / \mu \mathrm{L}$. They also observed significant difference between severity of the TCP and severity of the disease $(p$ value $=0.013){ }^{28}$

We observed that $68 \%$ patients were admitted with normal P-LCR, while $24.76 \%$ were seen with high P-LCR; only $7.14 \%$ were admitted with low P-LCR values. Mean P-LCR in DF (group I), DHF, and in DSS were $26.64 \pm 8.47,31.43 \pm 10.58$, and $32.80 \pm 9.28$ as we can see clearly from the observation table that P-LCR was increasing as the severity of the illness increased. High P-LCR was associated with longer duration of hospital stay and longer duration of fluid therapy requirement with a mean value of $4.96 \pm 3.26$ ( $p$ value 0.022 ) and $2.34 \pm 1.18$ ( $p$ value 0.289 ). Maximum patients who received ICU care were belonged to normal P-LCR group (28 patients/19.58\%) but out of total patients who were admitted with high PLCR $30.8 \%$ required ICU care. Patients with high PLCR were more commonly (28.85\%) landed up in shock, more frequently (26.92\%) given blood products, took longer time to recover from TCP with a mean value of $2.16 \pm 1.29$ days, and were associated with raised SGPT serum creatinine more commonly (75 and $19.23 \%$, respectively).

Bashir et al. conducted a study in Port Sudan, which found a median P-LCR of 31.13 (range 8.5-49.6\%); high P-LCR that correlated with TCP was observed in $15.6 \%$ of patients with DHF and $11 \%$ in DF patients. ${ }^{35}$

Ntaios et al. ${ }^{36}$ studied platelet indices (MPV, PDW, P-LCR) in idiopathic TCP purpura (ITP) and they found the PLCR values were low as compared to other TCP.

\section{Conclusion}

We found significant correlation of P-LCR, PDW, and MPV on admission with those morbidity parameters. High MPV, high P-LCR, high PDW, and low PCT were associated with longer duration of hospital stay, longer duration of fluid therapy given, longer time taken to recover from TCP, more frequent ICU care requirement, more frequent liver and renal function derangements, and they landed up in shock more frequently. Meticulous care by medical and paramedical staff, early recognition of danger signs and symptoms, and timely and necessary management helped in speedy recovery and prevention of mortality in these cases.

\section{Take Home Massage}

Careful monitoring in form of various clinical and laboratory parameters including different platelet indices (MPV, PLCR, PDW, $P(T)$ is very helpful in early identification, prognostication, and prevention of complications in dengue.

\section{Ethical Approval}

The study was approved by the institution ethics committee.

\section{References}

1. World Health Organization. Prevention and control of dengue fever and dengue hemorrhagic fever: comprehensive guidelines. WHO regional publication SEARO, No. 29. WHO; 1999.

2. Jayashree K, Manasa GC, Pallavi P, et al. Evaluation of platelets as predictive parameters in dengue fever. Indian J Haematol Blood Transfus 2011;27(3):127-130. DOI: 10.1007/s12288-011-0075-1.

3. Wiwanikit $V$. Mean platelet volume in the patients with dengue hemorrhagic fever. Platelets 2004;15(3):185. DOI: 10.1080/0953710042000199839.

4. Sachdev R, Tiwari AK, Goel S, et al. Establishing biological reference intervals for novel platelet parameters (immature platelet fraction, high immature platelet fraction, platelet distribution width, platelet large cell ratio, platelet-X, Plateletcrit, and platelet distribution width) and their correlations among each other. Indian J Pathol Microbiol 2014;57(2):231-235. DOI: 10.4103/0377-4929.134676.

5. Osselaer JC, Jamart J, Scheiff JM. Platelet distribution width for differential diagnosis of thrombocytosis. Clin Chem 1997;43(6 Pt 1):1072-1076. DOI: 10.1093/clinchem/43.6.1072.

6. Demirin $\mathrm{H}$, Ozhan $\mathrm{H}, \mathrm{Ucgun} \mathrm{T}$, et al. Normal range of mean platelet volume in healthy subjects insight from a large epidemiologic study. Throm Res 2011;128(4):358-360. DOI: 10.1016/j.thromres.2011.05.007.

7. Wiwanitkit V. Plateletcrit, mean platelet volume, platelet distribution width: Its expected values and correlation with parallel red blood cell parameters. Clin Appl Thromb Hemost 2004;10(2):175-178. DOI: 10.1177/107602960401000208.

8. Maluf CB, Barreto SM, Vidigal PG. Standardization and reference intervals of platelet volume indices: insight from the Brazilian longitudinal study of adult health (ELSA-BRASIL). Platelets 2015;26(5):413-420. DOI: 10.3109/09537104.2014.942620.

9. Vagdatli E, Gounari E, Lazaridou E, et al. Platelet distribution width: a simple, practical and specific. Marker of Mukker P et al. Int J Res Med Sci 2018;6(6):2054-2059.

10. Rasul CH, Mostafa KG, BaruriNN, et al. Comparative study of climate related target diseases in the coastal and plain area of southern Bangladesh. Malaysian J Public Health Med 2015;15(2):24-31.

11. Tripathi $P$, Kumar R, Tripathi $S$, et al. Descriptive epidemiology of dengue transmission in uttar Pradesh. Indian Pediatr 2008;45(4): 315-318.

12. Singla N, Chaudhary P, Thakur M, et al. Dengue: An analysis of epidemiological pattern over a six year period. J Clin Diagn Res 2016;10(12):DC12-DC14. DOI: 10.7860/JCDR/2016/22482.9011.

13. Shah I, Deshpande GC, Tardeja PN. Outbreak of dengue in Mumbai and predictive markers for DSS. J Tropical Paediatrics 2004;50(5): 301-305. DOI: 10.1093/tropej/50.5.301.

14. Agarwal A, Chandra J, Aneja S, et al. An epidemic of dengue hemorrhagic fever and dengue shock syndrome in children in Delhi. Indian Paediatrics 1998;35:727-732. 
15. Shivbalan $\mathrm{S}$, Anandanathan $\mathrm{K}$, Balasubramanian $\mathrm{S}$, et al. Predictors of spontaneous bleeding in dengue. India J Paediatrics 2004;71(1): 33-36. DOI: 10.1007/BF02725653.

16. Richard AL, Bagus R, Baso SM, et al. The first reported outbreak of dengue hemorrhagic fever in Irian Jaya, Indonesia. Am J Trop Med Hyg 1997;57(1):49-55. DOI: 10.4269/ajtmh.1997.57.49.

17. Halstead SB. Pathogenesis of dengue: challenge to molecular biology. Science 1988;239(4839):476-481. DOI: 10.1126/science.239.4839.476.

18. Malaviage G, Ranatunga PK, Velathanthiri VGNS, et al. Pattern of diseases in Sri Lanka dengue patients. Archive Disea Childhood 2006;91(5):396-400. DOI: 10.1136/adc.2005.085191.

19. Chacko B, Subramanian G. Clinical, laboratory and radiological parameters in children with dengue fever and predictive factors for dengue shock syndrome. J Trop Pediatr 2008;54(2):137-140. DOI: 10.1093/tropej/fmm084.

20. Chhina, Singh R, Goyal, et al. Liver function tests in patients with dengue viral infection. Dengue Bulletin 2008;32:110-117.

21. Itha S, Kashyap R, Krishnani N, et al. Profile of liver involvement in dengue virus infection. Natl Med J India 2005;18(3):127-130.

22. Kabra SK, Jain Y, Madhulika, et al. Role of platelet transfusion in dengue hemorrhagic fever. Indian Paediatric 1998;35(5):452-455.

23. Choudhary RR, Makwana M, Mourya HK, et al. Evaluation of platelet and its indices as a marker of neonatal sepsis: a prospective case control study. Int J Contemp Pediatr 2018;5(5):1898-1903. DOI: 10.18203/2349-3291.ijcp20183527.

24. Dewi1 R, Sukorini U, Intansari US, Correlation of enhancement Mean Platelet Volume (MPV) \& PDW with severity of dengue infection. 3rd Annual Applied Science and Engineering Conference (AASEC 2018).

25. Gunawan S, Sutantu FC, Tatura SNN, et al. Platelet distribution width dan mean platelet volume: hubungandengan derajat penyakit demam berdarah dengue. Sari Pediatri 2010;12(2):74-77. DOI: 10.14238/sp12.2.2010.74-7.
26. Maedel B, Doig K. Hematology clinical Principle and Applications Rodak B. 3th ed., USA: Saunders Elsevier; 2007.

27. Mukker $P$, Kiran S. Platelet indices evaluation in patients with dengue fever. Int J Res Med Sci 2018;6(6):2054. DOI: 10.18203/2320-6012. ijrms20182287.

28. Navya BN, Shwetha Patil TM, Kariappa Navya BN, et al. Role of platelet parameters in dengue positive cases - an observational study. Int J Health Sci Res (wwwijhsrorg) 2016;6(6):74-78.

29. Muddapu L, Kiran Kumar E, Kanchana PVN. Role of platelet indices in patients with dengue fever- a case control study. IOSR J Dent Med Sci 2019;18(2 Ser. 13):55-57.

30. Sharma K, Yadav A. Association of mean platelet volume with severity, serology \& treatment outcome in dengue fever: prognostic utility. J Clin Diagn Res 2015;9(11):EC01-EC03. DOI: 10.7860/ JCDR/2015/14285.6710.

31. Prakash GM, Anikethana GV. Use of mean platelet volume and platelet distribution width in predicting trend in platelet count and bleeding risks in patients of dengue fever. Int J Adv Med 2016;3(3):611-613.

32. Chiranth SB, Shreedhara Avabratha K. Platelet indices and the severity of dengue infection in children. J Pediatr Res 2019;6(3):242-246. DOI: 10.4274/jpr.galenos.2019.47568.

33. Xiaoyun L, Daowen W, Pengcheng X, et al. The changes and clinical significance of platelet parameters in children with dengue fever. Int J Laborat Med 2018;39(5):562-565.

34. Deshwal R, Ishaque Qureshi M, Singh R. Clinical and laboratory profile of dengue fever. J Associat Phys India 2015;63(12):30-32.

35. Bashir AB, Saeed OK, Mohammed BA, et al. Sudan role of platelet indices in patients with dengue infection in red sea state, Sudan. Int J Sci Res (IJSR) 2015. 4-438.

36. Ntaios G, Papadopoulos A, Chatzinikolaou A, et al. Increased value of mean platelet volume and platelet size deviation width may provide a safe positive diagnosis of idiopathic thrombocytopenic purpura. Acta Haematol 2008;119(3):173-177. DOI: 10.1159/000135658. 